\title{
High precision measurements of the neutron spin structure in HallA at Jlab
}

\section{John R.M. Annand, ${ }^{a}$, Todd Averett ${ }^{b}$, Gordon Cates, ${ }^{c}$, Evaristo Cisbani ${ }^{* d}$, Gregg B. Franklin $^{e}$, Nilanga Liyanage ${ }^{c}$, Andrew Puckett ${ }^{f}$, Guenther Rosner ${ }^{a}$, Bogdan Wojtsekhowski ${ }^{g}$, Xiaochao Zheng ${ }^{h}$}

${ }^{a}$ University of Glasgow, Glasgow G12 8QQ, Scotland, UK

${ }^{b}$ The College of William \& Mary, Williamsburg, VA 23185, USA

${ }^{c}$ University of Virginia, Charlottesville, VA 22904, USA

${ }^{d}$ INFN Rome - Sanità Group and Italian National Institute of Health, Rome, I-00161, Italy

${ }^{e}$ Cornegie Mellon University, Pittsburgh, PA 15213, USA

${ }^{f}$ Los Alamos National Laboratory, Los Alamos, NM 87545, USA

${ }^{g}$ Jefferson Lab, Newport News, VA 23606, USA

${ }^{h}$ Massachusetts Institute of Technology, Cambridge, MA 02139, USA

E-mail: john.annandeglasgow.ac.uk, tdaver@wm.edu, cates@virginia.edu, evaristo.cisbani@iss.infn.it, gbfranklin@cmu.edu, nl8n@virginia.edu, puckettejlab.org, g.rosner@gsi.de, bogdanwejlab.org, xiaochaoejlab.org

The $12 \mathrm{GeV}$ energy upgrade of the Continous Electron Beam Accelerator Facility of the Thomas Jefferson Laboratory (JLab), combined to high longitudinal polarization (up to 90\%), high current (up to $100 \mu \mathrm{A}$ ) and new experimental equipments has stimulated the definition of several physics proposals aimed at the precise measurements of the nucleon structure observables in unexplored kinematics regions.

Two of these experiments, expected to run in Hall A, the largest JLab experimental hall, intend to exploit a new ${ }^{3} \mathrm{He}$ polarized target and new detectors to investigate Deep Inelastic Scattering processes at high luminosity, in the high $x$-Bjorken region. For the first time, fundamental quantities such as the $A_{1}^{n}$ asymmetry and different transverse spin and momentum dependent quark distribution functions will be measured with high precision in the quark valence region.

In this report, theoretical and experimental details of the above experiments will be shortly presented.

Sixth International Conference on Quarks and Nuclear Physics

April 16-20, 2012

Ecole Polytechnique, Palaiseau, Paris

\footnotetext{
*Speaker.
} 


\section{Introduction}

The long standing quest for the origin of the spin of the nucleon, after the last couple of decades of exciting discoveries, is going to take a remarkable leap at the Thomas Jefferson National Laboratory in the coming years.

JLab is undergoing an important upgrade which will double the maximum available energy of its high intensity longitudinally polarized electron beam by the beginning of 2014 . The beam will be delivered simultaneously on the 3 original experimental halls (A, B and C adequately upgraded) and a new hall (D) dedicated to real photon physics. An intense physics program will exploit the advantages offered by the new JLab experimental equipment to investigate the nucleon spin structure, in terms of high precision and extended phase space.

In this respect, high luminosity experiments will be carried on in the Hall A on a polarized ${ }^{3} \mathrm{He}$ target (effective neutron target) in the Deep Inelastic Scattering (DIS) limit; two of them will be shortly described in the following sessions: an inclusive measurement with unprecedented precision [1] will be devoted to the determination of the photon asymmetry $A_{1}^{n}$ at large $x$-Bjorken $\left(x_{B j} \sim 0.71\right)$, in the valence region. In addition, the first high statistics determination of the neutron single spin asymmetry in semi inclusive scattering of pion and kaon off transversely polarized ${ }^{3} \mathrm{He}$ target [2] is also expected to run in Hall A, using the newly developed high luminosity, large acceptance Super BigBite Spectrometer (SBS) [3].

\section{Physics motivations and goals}

The current picture of the nucleon structure grounds essentially on three related quantities [4]: the nucleon form factors, the parton distribution functions (PDF), and the generalized parton distribution functions (GPD). All of them can be derived from the phase-space parton distribution Wigner functions introduced in [5]; according to the present understanding the Wigner functions cannot be measured directly.

Form factors, PDFs and GPDs are related to fundamental properties of the nucleon and its internal dynamics, such as the origin of the spin and the orbital angular momentum (OAM) of its constituents, and therefore to the complex, and largely unknown, aspects of the QCD.

In recent years, investigation on the spin and transverse momentum dependent PDFs has triggered significant experimental and theoretical advances. PDFs are mainly accessed by the measurement of the cross section asymmetries on polarized beam and/or target, in DIS (inclusive and semi inclusive, SIDIS) and, in Drell-Yan processes (assuming some sort of PDF universality is valid).

Of the 8 leading twist PDFs, five are transverse momentum dependent (TMD) distribution functions expressing the probability of the quark with transverse momentum $\left(k_{T}\right)$ and a given polarization in a nucleon with defined polarization relative to the direction of the nucleon. The remaining 3 PDFs are the well known unpolarized distribution $f_{1}(x ; Q)$, the rather known helicity $g_{1}(x ; Q)$ and the recently measured transversity $h_{1}(x ; Q)$ being $x$ the momentum fraction of the quark relative to the momentum of the nucleon and $Q$ the energy scale (momentum transfer in DIS). The first moment $\int_{0}^{1} d x g_{1}(x ; Q)$ is directly related to the quark spin contribution to the total spin of the nucleon. On the other hand $h_{1}$ presents peculiar features: it is expected to be identical to $g_{1}$ in a 
non relativistic limit; gluons do not contribute to its $Q^{2}$ evolution and its first moment is the tensor charge of the nucleon.

The two proposed experiments $A_{1}^{n}$ and SIDIS will access 6 of the 8 PDFs in electron scattering experiments off a polarized ${ }^{3} \mathrm{He}$ target (an effective neutron target) in DIS: helicity, transversity, Sivers $f_{1 T}^{\perp}\left(x, k_{T} ; Q\right)$, pretzelosity $h_{1 T}^{\perp}\left(x, k_{T} ; Q\right)$ and the worm-gear $g_{1 T}\left(x, k_{T} ; Q\right)$. While helicity and worm-gear can be measured by inclusive double spin asymmetries (polarized target and beam), the other PDFs need an additional particle in the final state (semi-inclusive) with transversely polarized beam and are therefore convoluted with fragmentation functions.

Both Sivers and worm-gear are connected to the quark OAM; in addition the sign of the Sivers in SIDIS is expected to be opposite to the one measured in Drell-Yan, according to fundamental QCD gauge principles [6]; $g_{1 T}$ presents one of the first attempt of lattice QCD calculation. The pretzelosity somehow measures the deviation of the nucleon from a spherical shape, and naively (model dependent) is the difference of the helicity and transversity PDF's.

While there are clean evidence of signal for the transversity and Sivers PDFs, the modest measurements of the pretzelosity are consistent with 0 , while the first worm-gear estimations give evidence of possible non zero values.

All measurements, with few exceptions, lack precision, have limited coverage of phase space (in particular at high $x_{B j} \sim x$, where signal may be larger), and are integrated on two of the three relevant kinematics variables $\left(x, z\right.$ or $\left.p_{T}\right)$; on the other hand the experimental knowledge of $g_{1}$ (and its related asymmetry $A_{1}$ ) is rather detailed, however data at high $x_{B j}$ is missing (fig. 1). All these limitations are more evident in the neutron, preventing, in most of the cases, an unambiguous interpretation of the data.

In this context, the $A_{1}^{n}$ experiment aims at a precise measurement of the $A_{1}^{n}$ asymmetry in DIS, in the range $\sim 0.3<x_{B j}<0.71$, and, in combination with new data on the proton, will also permit a clean flavor decomposition of $g_{1}$. The asymmetry $A_{1}$ is calculable in perturbative QCD (pQCD), in the valence region, at high $x$, where the contribution from the sea partons is expected to be marginal. The pQCD calculations are sensitive to the OAM and therefore the measure of $A_{1}$ at high $x$ provides important insight into the role of the quark OAM in the nucleon.

On the other hand, the SIDIS experiment, in the line of the recently completed E06-010 experiment [8], intends to measure, for the first time, with high precision, providing 3D binning on the relevant kinematics variables (and $Q^{2}$ dependence), the semi-inclusive transverse target single spin asymmetry, a linear combination, of the Collins, Sivers and the pretzelosity asymmetries, convolution of $h_{1}(x ; Q) \otimes H_{1}^{\perp}\left(z, p_{T} ; Q\right), f_{1 T}^{\perp}\left(x, k_{T} ; Q\right) \otimes D_{1}(z ; Q)$ and $h_{1 T}^{\perp}\left(x, k_{T} ; Q\right) \otimes H_{1}^{\perp}\left(z, p_{T} ; Q\right)$ respectively, where $H_{1}^{\perp}$ and $D_{1}$ are the Collins and unpolarized fragmentation functions and $z$ and $p_{T}$ the fractional energy and transverse momentum of the detected hadron. Moreover the SIDIS experiment will extract the double spin longitudinal beam/transverse target asymmetry related to the worm-gear PDF.

\section{Experimental setup}

The $A_{1}^{n}$ and SIDIS electron scattering experiments, in the DIS region, share basic experimental ingredients: a new $3 \mathrm{He}$ polarized target, large acceptance dedicated spectrometers and high quality beam. 

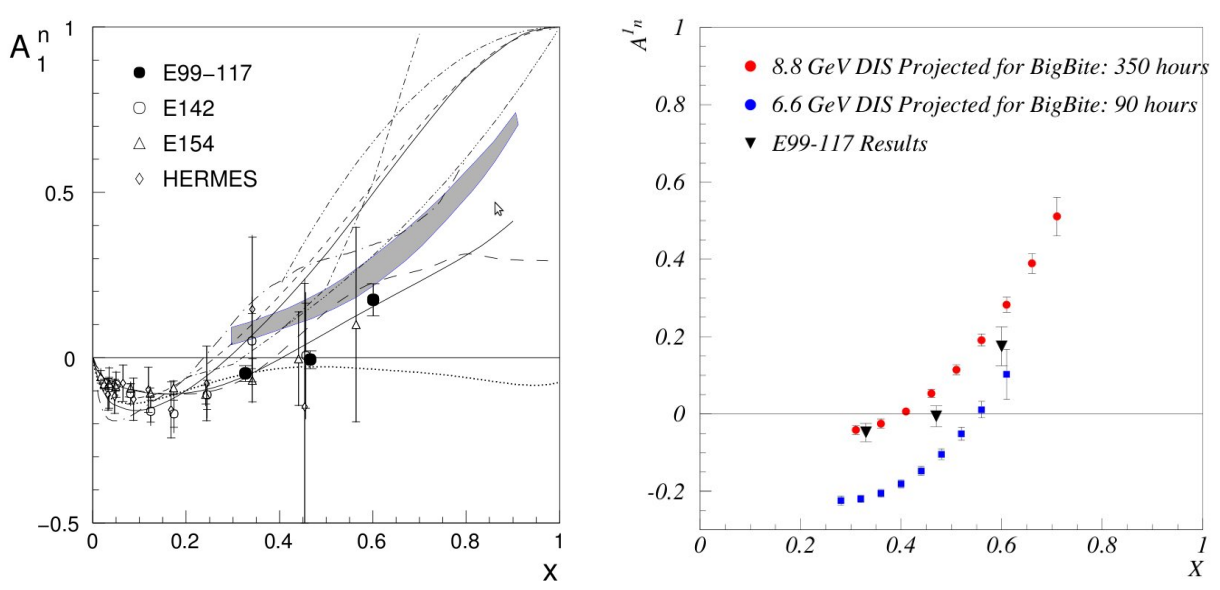

Figure 1: Left: $A_{1}^{n}$ measurements and different theoretical predictions; refer to fig. 1 of the $A_{1}^{n}$ proposal [1] for details. Right: $A_{1}^{n}$ projected statistical errors compared to the last JLab measurement (E99-117 results).

The existing polarized ${ }^{3} \mathrm{He}$ target of HallA is based on laser driven optical pumping of $\mathrm{Rb}-\mathrm{K}$ vapor with spin exchange by the ${ }^{3} \mathrm{He}$ hyperfine interaction; the ${ }^{3} \mathrm{He}$ polarized gas diffuses into a glass tube that connects the pumping chamber to the target cell. This target has demonstrated a steady operation at $65 \%$ polarization at $10 \mu \mathrm{A}$ beam current [7]. The new target, under development at the University of Virginia, will be based on the same mechanism of ${ }^{3} \mathrm{He}$ polarization but the gas transfer to the target cell will be improved by convection on two tubes and the target cell will be coated by metal window to support higher beam current. Adiabatic spin flip and holding magnetic field system will be able to provide the different polarization directions required for both experiments (up to 8 transverse directions, in the SIDIS experiment, to uniformly cover the azimuthal phase space).

The $A_{1}^{n}$ experiment will exploit the BigBite dipole spectrometer, successfully used in recent DIS experiments as electron arm [8]. The SIDIS experiment in coincidence with the electron in BigBite will detect a pion or kaon in the new SBS, large acceptance, spectrometer. Both spectrometers will be equipped with trackers able to support high background rate up to $250 \mathrm{MHz} / \mathrm{cm}^{2}$ photons. These trackers are based on the GEM technology [9] providing hit spatial resolution at the level of $70 \mu \mathrm{m}$, that combined to $\sim 1.2 \mathrm{Tm}$ magnetic field integral, will correspond to momentum resolution $\delta p / p<1 \%$, angular resolution of $\sim 0.5 \mathrm{mrad}$ and vertex resolution of $\sim 1 \mathrm{~mm}$.

Both experiments will run in high luminosity conditions $\left(\sim 10^{37} / \mathrm{cm}^{2} / \mathrm{s}\right)$ with beam current up to $50 \mu \mathrm{A}$ and high (up to $90 \%$ ) longitudinal polarization. Although not required for the extraction of the single spin asymmetry in the SIDIS experiment, the longitudinal polarization will be exploited to measure, at the same time, double beam and target spin asymmetry related to the worm-gear $g_{1 T}$. Two energy beam settings, in both experiment will permit an adequate coverage of the $Q^{2}$ region from 2 to $9 \mathrm{GeV}^{2}$ ( $Q$ is strongly correlated to the $x_{B j}$ ).

The SIDIS experiment will re-use, as pion-kaon identification, one of the HERMES RICH detectors, in the populated momentum range $(\sim 2-8 \mathrm{GeV} / \mathrm{c})$. Time information of the detected Cherenkov photons, will be used to reduce the occupancy below $1 \%$, imposing an offline time window of $10 \mathrm{~ns}$. 

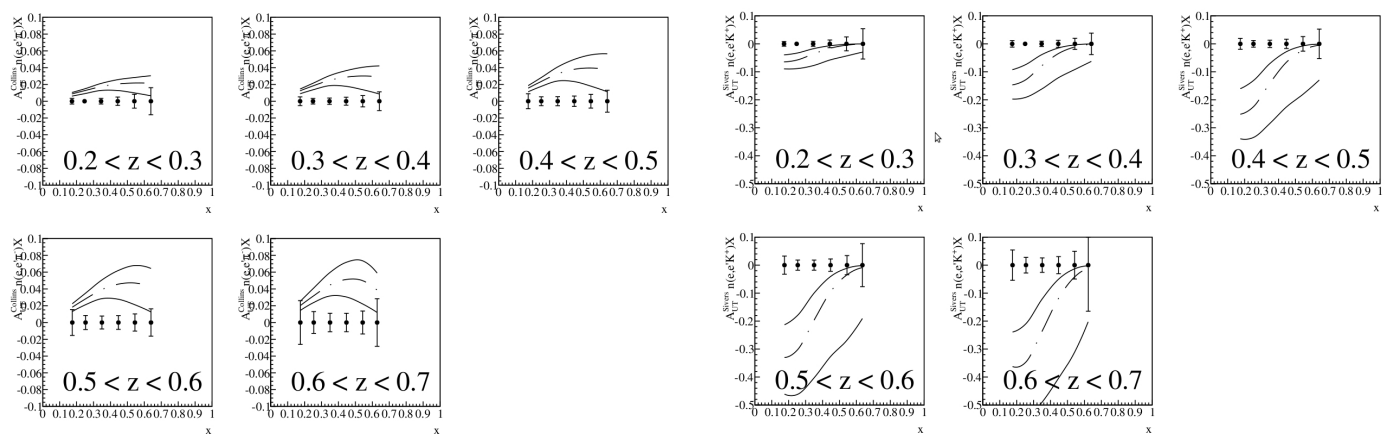

Figure 2: Expected precision in the SIDIS experiment, as a function of $x_{B j}$ for different $z$ slices for the $\pi^{-}$ Collins asymmetry (left) and the $K^{+}$Sivers asymmetry (right); curves are global fits on existing data [10] with central value and uncertainty corridor.

\section{Conclusions}

$A_{1}^{n}$ experiment will be likely one of the earlier experiments at JLab after the energy upgrade. Besides the importance on its own, the experiment will represent a test for the new generation high luminosity experiments devoted to the nucleon structure investigation that will characterize large part of the experimental activity in Hall A.

Both $A_{1}^{n}$ and SIDIS experiments will provide new results in Deep Inelastic Scattering electronneutron processes in unexplored phase space regions (e.g. in the valence region) with unprecedented statistical errors, which imposes a well understanding of the source of systematics uncertainties.

\section{References}

[1] JLab Experiment E12-06-122 ( $\left.A_{1}^{n}\right)$ : http://hallaweb.jlab.org/collab/PAC/PAC30/PR12-06-121-A1n.pdf

[2] JLab Experiment E12-09-018 (SIDIS): http://hallaweb.jlab.org/collab/PAC/PAC38/E12-09-018-SIDIS.pdf

[3] Super BigBite Spectrometer web page: http://hallaweb.jlab.org/12GeV/SuperBigBite/

[4] M. Burkardt, A. Miller and W-D. Novak, Rep. Prog. Phys. 73 (2010) 016201

[5] A.V. Belitsky, Xiangdong Ji, Feng Yuan, "Quark Imaging in the Proton Via Quantum Phase-Space Distributions”, arXiv:hep-ph/030738v1, 31 Jul 2003

[6] J. C. Collins, Phys. Lett. B536 (2002) 43

[7] Jin Huang, "Double Spin Asymmetry $A_{L T}$ in Charged Pion Production from Deep Inelastic Scattering on a Transversely Polarized ${ }^{3} \mathrm{He}$ Target", PhD Thesis, MIT, Feb. 2012, web link: http://hallaweb.jlab.org/experiment/transversity/thesis/Huang_thesis.pdf

[8] X. Qian et al., Phys.Rev.Lett. 107 (2011) 072003

[9] F. Sauli, Nucl. Instr. and Methods A386 (1997) 531

[10] M. Anselmino et al., Phys. Rev. D75, 054032 (2007); M. Anselmino et al., EPJ A 39, 89 (2009) 